\title{
Microblogging for Language Learning: Using Twitter to Train Communicative and Cultural Competence
}

\author{
Kerstin Borau ${ }^{1}$, Carsten Ullrich ${ }^{2}$, Jinjin Feng ${ }^{1}$, and Ruimin Shen ${ }^{2}$ \\ ${ }^{1}$ Distance Education College of Shanghai Jiao Tong University, \\ 3/F No. 500 Jiangsu Road, Shanghai 20050, China \\ ${ }^{2}$ Shanghai Jiao Tong University, 1954 Hua Shan Road, 200030 Shanghai, China \\ kerstinborau@mail.online.sjtu.com, \\ \{ullrich_c,jjfeng,rmshen\}@sjtu.edu.cn
}

\begin{abstract}
Our work analyzes the usefulness of microblogging in second language learning using the example of the social network Twitter. Most learners of English do not require even more passive input in form of texts, lectures or videos, etc. This input is readily available in numerous forms on the Internet. What learners of English need is the chance to actively produce language and the chance to use English as tool of communication. This calls for instructional methods and tools promoting 'active' learning that present opportunities for students to express themselves and interact in the target language. In this paper we describe how we used Twitter with students of English at the Distant College of Shanghai Jiao Tong University. We analyze the students' messages and show how the usage of Twitter trained communicative and cultural competence.
\end{abstract}

Keywords: ESL, microblogging, language learning, communication.

\section{Introduction}

Most learners of English do not require even more passive input in form of texts, lectures or videos, etc. - they need a chance to actively produce language and the chance to use English as tool of communication. This calls for instructional methods and tools promoting 'active' learning that present opportunities for students to express themselves and interact in the target language. Such an opportunity is offered by social networks with English as the dominating language.

The following paper shows how the social network Twitter (http://twitter.com) was applied in an EFL (English as a Foreign Language) classroom at the Shanghai Jiao Tong Distance College (Online-SJTU) for seven weeks and two days in the summer term 2007. During the course, about 90 students made a total of almost 5580 updates - a rich dataset we analyzed for different indicators from a language learning perspective. To our knowledge, this is the first analysis of microblogging usage for EFL.

Twitter was selected because it provides the Chinese students the opportunity to practice the target language as well as their communicative and cultural competence. Most Chinese students are able to express their transactional intentions, but according to our experience, they have great difficulty to do this on a socio-linguistic acceptable 
level. Twitter has a huge advantage over usual discussion boards: Tweets consist of short turns including no more than one or two utterances. Discussion boards usually require the participants to create and follow up on a thread or to follow a thread and comment. This requires long turns which are significantly more demanding [2]. Since the creation of long turns is much more complicated and far more time consuming than the production of short turns found in tweets, it was decided that Twitter is more suitable for the language level and limited study time available to our students.

The paper is structured as follows. After introducing the setting of our Twitter case study in Section 2, we analyze the data in: the first part (Section 3) explores the use for language learning, namely the training of aspects of communicative competence as well as cultural competence. The second part (Section 4) analyses the student community engaged in Twitter as a blended learning community and points out the positive influence on motivation. Additional results are reported in Section 5. Section 6 discusses related work and provides a conclusion, including links to our datasets.

\section{Description of the Case Study and the Datasets}

\subsection{Twitter and How It Was Used in the Course}

Twitter is a social network in which the members of a community share their current activity by answering the question "What are you doing?". This form of social interaction is called microblogging. Microblogging tools enable users to post short messages that are distributed within their community. Users can post messages from their mobile devices, a Web page, from Instant Messengers and desktop clients. The same channels are used for receiving messages.

Twitter seems to be a perfect tool to support learning English, especially in blended classrooms, for the following reasons. First, Twitter is easily accessibly from almost everywhere, so the students can practice at any time by sending and receiving messages either on the computer or the mobile phone. They can also decide how much time they spend reading and writing these messages. This is an essential feature for busy students. Second, Twitter is suitable for any level of English because in writing the messages, the students can chose a topic and grammatical structure fitting their level. Third, the use of Twitter as an online learning community can help to integrate students in the community who could not attend classroom.

In this work, the microblogging tool Twitter was used in an English course for native speakers of Chinese in the following way: the instructor created a new, personal Twitter account. As homework, the students of this class were prompted to create their own account and to become "friends" with the instructor's account as well as with the accounts of the other students. Since each Twitter user receives the messages of his or her friends, each student who followed the instruction would receive the messages of his/her fellow students and of the instructor. The students were then told to post at least seven microblogging messages a week and to read the incoming messages of their fellow students. 


\subsection{Students and Study Context}

The students at the Online-SJTU attend blended classrooms, that is, students can attend in person or virtually using their browser to watch a live stream of the lecture. Our blended classrooms are based on the Standard Natural Classroom model [20] providing face-to-face interaction with the instructor as well as online courses.

There are three main problems occurring in the English courses at the OnlineSJTU: The limited time available for study, the different levels of English, and the size of the classes: the students at the Online-SJTU are adult learners which come from varying social and educational backgrounds. Consequently, there are also huge variations in their English levels. Some have been studying English continuously during the last 10 years while others have just started learning English in evening classes. Most of the students have a demanding full time job and hence do not have an hour of idle leisure time which they can fill with long-winded excises. Still, they spend time commuting or might have slack minutes in the office which they might as well spend learning and practicing their English skills.

The class sizes at the Online-SJTU vary from 80 to 120 students; thus not everybody has the chance to communicate with the instructor during class and practice his or her English skills.

\subsection{The Datasets}

\section{The Updates}

Twitter was introduced in June 2007 in the lecture "English Listening \& Speaking" which aims at improving the students' communicative skills in English. In order to increase Twitter participation, participation in Twitter contributed to the students' final grade. During the period relevant for grading, the students' updates were counted each week and a score was assigned. The score did only depend on the amount of messages and did not take into account the linguistic correctness of the updates. To give students time to register at Twitter and to get familiar with its features, the grading period started two weeks after Twitter was introduced.

From the 110 students enrolled in the lecture, a total of 98 students registered at Twitter. Of the 98 registered students, about 10\% (12 students) never used Twitter. One third (35 students) sent between 1 and 19 updates, again one third (39 students) sent 20 to 99 updates, and about 10\% (12 students) sent more than 100 updates (10 students sent between 100 and 162 updates, and 2 students dominated the Twitter updates with 449 and 236 updates, respectively.

The dataset we analyzed covers a total of seven weeks and two days. During that time, students sent a total of 5574 updates, which corresponds to an average of 796 updates a week or 113 per day.

\section{The Questionnaire}

At the end of the lecture, students were asked to fill out a bilingual questionnaire (English/Chinese) about their usage of and opinion on Twitter. A total of 96 students completed the questionnaire. Of these, 82 students claimed to have used Twitter at least once. Since this paper investigates the effects of usage of Twitter, we excluded the 14 questionnaires of the students who never used Twitter from the analysis. 
The questionnaire further showed that most participants $(62=75 \%)$ of the students who completed the survey were female, $25 \%(=20)$ were male. Half of the participants were aged 20-25 (=42), 40\% (=33) were aged $25-30$, and $10 \%(=7)$ over 30 .

\section{Twitter and Language Learning: Training Communicative and Cultural Competence}

\subsection{Using Twitter in Training Communicative Competence}

In our project, Twitter was viewed as a supplement to practice in authentic environment different aspects of the target language as it was taught in the classroom. One aspect of language learning which can be practiced in Twitter is communicative competence [13], a concept based on a view of language learning postulated in the communicative approach to language teaching, in which interaction is focused as a means of language learning and teaching.

Current views on language teaching emphasize communicative competence as the main goal of language learning (e.g. [19]). According to [3], communicative competence consists of four components: grammatical competence (words and rules); sociolinguistic competence (appropriateness), discourse competence (cohesion and coherence), and strategic competence (appropriate use of communication strategies).

In this paper, we will focus on two subcategories of communicative competence: sociolinguist and strategic competence. Further, we restricted the terms sociolinguist and strategic competence according to our purposes of our analysis.

\section{Sociolinguistic Competence}

We define sociolinguistic competence as the ability to use and respond to language appropriately, with regard to the setting, the topic, and the relationships among the community. We studied the students' updates with regard to their ability to express a specific attitude (courtesy, friendliness, annoyance). The updates show that most of the students have problems to choose the appropriate style and / or maintain one level of style. The following example illustrates the mixture of styles by using a more formal expression, "to pursue", and a rather colloquial expression, "wanna" (in the remainder of the article, all indented italic sentences are updates sent by the students):

"Richard suggested me chaning my life and pursuing the life I wanna."

The overall atmosphere on Twitter was polite and friendly; consequently the main style was in general colloquial. However, within the single updates students chose expressions of a rather formal style, such as "due to" in the first example and "I take responsibility" in the second, which is inappropriate for the purpose of communicating in an online community:

"Due to there will no classes on August, so I made a plan to visit Hongkong with my family."

"So I take the responsiblity of buying vegetable."

Other students sent updates in slang-like style spiked with swear words such as "damn" and "screw up", which is also inappropriate for the community:

"Damn exams, I hate it, A LOT! However, I don't wanna scew up on it." 
"sleeping in the internet bar about 3 hours.Too damn uncomfortable.. take a bath about a.m 5:30...soon $i$ will go to work... a day of unlucky"

Within the data, a large number of similar examples show that using a microblogging tool in a course focusing on communication provides numerous opportunities to practice sociolinguistic competence. The teacher should take up the these utterances and discuss them in class, to help the students understand their (in)appropriateness.

\section{Strategic Competence}

Strategic competence refers to the ability to handle communication breakdowns. In the following, we focus merely on how the students react if they don't know the name of something or the right verb form to use. In these examples, the students didn't know the correct name to refer to something, and thus used the Chinese word:

“'普洱茶' is useful to lose weight. At least,it helps me a lot. Sorry, I don't know how to say it in English”

“桂林米粉 how to say in English?”

Judging from the update data, this strategy, which is also referred to as appeal for assistance, is utilized only in few cases. To further investigate strategic competence outside the Twitter updates, a series of questions inquired about usage of tools such as (online) dictionaries. When asked whether they use a dictionary for writing messages, a vast majority of $69.51 \%$ reported that they sometimes use a dictionary. $15.85 \%$ stated they never used one, and $14.63 \%$ stated that often used one. The figures for reading messages were similar, $67.07 \%$ sometimes, $19.51 \%$ never and $13.41 \%$ often used a dictionary. The utilization of dictionaries and the limitation to 140 characters in the updates partly explain the lack of using communication strategies in case of communication breakdowns; we therefore conclude that Twitter in itself is of no great help in practicing strategic competence.

\subsection{Using Twitter in Training Cultural Competence}

Cultural competence is a subcategory of sociolinguistics [7]. It consists of four categories: awareness of one's own cultural worldview, attitude towards cultural differences, knowledge of different cultural practices and worldviews, and cross-cultural skills. Cultural competence helps people from different parts of the world to effectively interact across cultures. In Twitter, the interaction with native speakers of English and the usage of English speaking Web-services helps to create a sense of cultural awareness and acquiring cultural competence in addition to English skills, as a student noted in the free answer section of our questionnaire:

"I also can learn some characteristic sentences by reading some native speaker's messages."

There is a need for training cultural competence with regard to Internet usage, as the following quote from the questionnaire's free answers section supports:

"Twitter is a foreign chating network.It is hard for Chinese people to link it and update messages."

According to the survey, the participants frequently use the Internet. $90 \%$ use it daily, $10 \%$ weekly. However, most of time, they visit Chinese Web sites. $37 \%$ of the students visit mostly Chinese Web sites, 57\% more Chinese than English Web sites, 
4\% more English than Chinese Web sites and 2\% mostly English Web Sites. Additionally, a large number of students stated in the questionnaire that they are in an allChinese environment and hardly ever communicate in English in their daily lives, which makes training cultural competence especially difficult.

In the questionnaire, almost half of the students stated that they communicated with native speaker on Twitter. However, it is difficult to confirm this data by analyzing the actual updates. Only few updates contain clear evidence that the updates were addressed to a person outside the class, for instance by stating the recipient's user name. Due to privacy restrictions, we were unable to use the follower list as an additional source of information. Still, if we believe the survey, students recognized Twitter as an opportunity to communicate with native speakers.

Of course cultural competence in its full scope cannot be acquired by simply joining a $\mathrm{SN}$, but it can provide an insight into another culture and create an awareness of existing differences. Twitter allows the students to get in contact with people from different countries on a casual basis and thus provides cultural background to the target language. The awareness of different cultural backgrounds also includes an awareness of different communication strategies which become evident during interaction. Using Twitter, the students have the opportunity to communicate and interact more effectively with people across cultures; a skill which becomes more and more indispensable in the interconnected world of today.

\section{Motivation through Community}

\subsection{Twitter as a Blended Learning Community}

Blended Learning Communities are groups which utilize face-to-face meetings as well as online meetings. The social coherence and social interaction provided in online learning communities enable the exchange of information, and they further provide part of the motivation for the learners because the sense of belonging to a community of learners motivates the individual to conform to the group learning behaviors.

Evidence of a Sense of Community among the Participants in the Twitter Project There is evidence that the participants in the Twitter-project indeed formed a community, first of all from the questionnaire. When asked whether Twitter improved the sense of community in the class, $40 \%$ completely agreed, about at third somewhat agreed, $23 \%$ were neutral, and 5\% (= 4 students) disagreed.

Additionally, a number of linguistic markers for socializing can be found, such as greetings and group reflective observation, that is, any kind of comment on the group or its identity [15], and we also find humorous updates. A few examples follow.

A number of students visited Twitter in the morning before they started work, so we find different greetings which are variations of good morning:

"Good morning everyone."

"Morning!"

The students also visited Twitter before they went to sleep:

"So tired today, and going to bed now. Hope everybody ave a sweet dream tonight. See u tomorrow." 
"Time to sleep now, have a good night and sweet dream to everyone!" They also commented on the community in different updates. One update relates to the avatars used by the participants of the project:

" $i$ just found something is funny, why everybody like pets in our class?for example:dog,cat and others."

Another student comments directly on the community:

"This "TWITTER" same as schoolyard "

Humor as a marker for the forming of a community can also be observed in the updates. When the instructor updated that she was busy proofreading the exam papers for this course, a student jokingly offered his help:

“@ kerstinlaoshi I volunteer to proofread the exam paper for you!”

Still, another interesting aspect of socializing is presented by the conversations in Twitter, which is described in the following paragraph.

\section{Conversations in Twitter as Makers of Community}

First of all, Twitter is not designed for conversations, but rather for updates ('I'm having lunch now"); still people engage in conversations $[11,18]$, which are markers of social coherence and community forming. In [15], Kellogg classified several features of conversations; in the following we will focus on what she designates as $R e$ sponse and Elaboration. According to Kellogg, Response includes responding directly to questions or points raised previously while Elaboration refers to elaborating on points raised earlier by oneself or others. The two latter features occur in conversation using the @ symbol. In [14] the authors pointed out that "since there is no direct way for people to comment or reply to their friend's posts, early adopters started using the @ symbol followed by a username for replies". However, Elaboration also occurs without the @ symbol and is only indicated by the closely related content of the updates referring to own or others' updates. The second type (no@ symbol) contains more general comments on topic, while the first type (with@ symbol) is used to make a specific statement or clarify a specific point.

The following illustrates a short but typical @-conversation about online shopping:

[15:21] babyyao: @Nora_Tang:I would buy some cosmetics online these days.

[15:55] Nora_Tang: @babyyao I bought 4 books online just now. The most important thing is that the goods(shopping online) are cheaper.

Below is an example of a short conversation without @ in which the updates are related through the topic "film":

[09:13] icyhe: Transformer is very nice. i like it. Funny!

[09:36] misterjustin: $i$ like the "bumble bee"best

Of course these two features of conversations occur fused within in the data. For instance, the conversation on "Transformers" is longer and also contains responses with the @ symbol as well as elaborations; such as the following update, which actually triggered the updates relating to "Transformers" and movies in general:

“@kerstinlaoshi:so, how's the transformer? Was it as good as the ads?”

The questionnaire also investigated conversations. One third of the students stated that they often replied to updates of their classmates. More than half $(56 \%)$ sometimes 
and only $14 \%$ never replied to updates. The updates confirm these numbers. In total, about $8 \%(=447)$ of the updates contained the "@” symbol.

Conversations implicitly provide motivation through social support by giving the individuals the sense of belonging to a group. In our data there are also conversations which provide social support explicitly in making it the topic of the conversation:

"maybe they are busy now. Don't worry! If you have some problems, you can contact with $m e^{\wedge} 0^{\wedge}$."

\section{Additional Results}

In this section, we will briefly describe additional results from the questionnaire relevant for this paper, such as frequency and length of visits.

Three questions investigated the average frequency of Twitter visits and the time spent on the site. A majority of the students frequently interacted with Twitter. On average, one quarter $(25.61 \%)$ stated they visited the site at least several times day. Again one quarter $(25.61 \%)$ visited Twitter once a day. $39.02 \%$ went to the site less than once a day but still several times a week. $3.66 \%$ students visited it once a week, $4.88 \%$ less than once a week.

More than two third of the students spend little time to write a Twitter update. $31.71 \%$ spent less than 1 minute, $36.59 \%$ between 1 and 2 minutes. About one quarter (23.17\%) spent 2 to 5 minutes. $7.32 \%$ invested 5-10 minutes, and only a single student $(1.22 \%)$ spent more than 10 minutes. These figures substantiate the claim that Twitter serves as a quick and easy medium for informal communication.

A similar pattern was visible in the reading behavior. When asked how much time the students spent reading Twitter updates on an average visit, about $95 \%$ spent less than 15 minutes. More specifically, 26.83\% spent less than 5 minutes, $45.12 \%$ from 5 to 10 minutes, and $23.17 \%$ between 10 to 15 minutes. Only $4.88 \%$ (4 students) spent between 15 to 30 minutes. No student spent more than 30 minutes reading updates. This data supports our expectation that Twitter is a tool that is frequently accessed for a short time span, with a short amount of time spent writing updates and a bit longer but still limited time spent reading the classmates' updates.

The effects of Twitter usage on English communication as perceived by the students were investigated by two questions. About $70 \%$ of students stated that they find it easier to communicate after using Twitter (30\% completely agreed, $40 \%$ somewhat agreed). About one quarter $(24 \%)$ had a neutral opinion and $4.88 \%(=4$ students) disagreed. No student completely disagreed. When asked whether they felt less shy when communicating in English after their Twitter usage, a similar but slightly less positive distribution arouse with $28 \%$ completely and $34 \%$ somewhat agreeing. $31 \%$ were neutral and $6 \%$ somewhat disagreed. Again, no student completely disagreed. All in all, these figures show a positive view on the effects of Twitter with respect to communication. Interestingly, the numbers of students disagreeing (4 and 5) was smaller than the students who sent no updates at all (12, as taken from the log data). This might be caused by the students seeing a "theoretical" value in using Twitter. 


\section{Related Work and Conclusion}

Related work on microblogging covers either general suggestions on how to use Twitter in the classroom or analyses properties of the network and conversations without a focus on learning. In the former category, general hints and advice for academic usage have been published in several blogs (e.g., [1, 4, 17]), Websites (e.g., http://twitterforteachers.wetpaint.com) and publications [6, 8, 21]. [10] describe how they used a Romanian microblogging service to deliver an online course.

From an analytical viewpoint, the first published analysis of Twitter [14] reports on topological and geographical properties, and also describes the most frequent usage types (daily chatter, conversations, sharing information and reporting news). [16] analyzes a broader subset of Twitter users. In addition to information about its user base in general and its geographical distribution, they identify different users types based on the amount of followers, friends and update frequency. The specific kind of communication that takes place on Twitter is analyzed in [11, 18]. [18] shows that communication on Twitter goes beyond updating one's status messages. Most tweets address specific friends. This is confirmed by [11] who give a detailed analysis of the usage of Twitter as a conversation tool. A similar analysis is done in [12]; however the authors identify an inner, much smaller network of friends. Suggestions for analyzing microblogging are listed in [9]. Probably the only work that analyzes Twitter in the context of learning is [5]. They performed a frequency analysis over Twitter updates collected during an intensive one week summer school for $\mathrm{PhD}$ researchers. The students were instructed to use Twitter a back-channel for communication. In their data the most frequent keywords were directly related to the terms used in the summer school lectures, which indicates that the students were using Twitter as instructed.

The suitability of Twitter for language learning was often postulated, but to our knowledge until now never investigated in detail. Our work explores Twitter as a tool for active learning in the EFL classroom and provides the first detailed analysis of how Twitter was used to learn specific aspects of language. Our main finding is that it is suitable to train communicative and culture competence anytime anywhere without face-to-face interaction. In a broader scope, this work contributes to the research on using Web 2.0 services and tools for learning, so called e-learning 2.0. We show that the social, collaborative principles of Web 2.0 are reflected in its usage by learners, if used in the appropriate way. In our case, students clearly like using the service; one student even asked to continue to use Twitter after the project: "Would you please do not close the twitter online? It offers us a free space to put ourself in English."

To enable further research by interested parties, we made the dataset available as a free download on the Website "Many Eyes", a data visualization service by IBM. (http://manyeyes.alphaworks.ibm.com/manyeyes/users/ullrich).

\section{References}

[1] Al-Khalifa, H.S.: Twitter in academia: a case study from Saudi Arabia. eLearn. 2008(9), $1-1$ (2008)

[2] Brown, G., Yule, G.: Teaching the spoken language. Cambridge Language Teaching Library (1999)

[3] Canale, M., Swain, M.: Theoretical bases of communicative approaches to second language teaching and testing. Applied Linguistics 1, 1-47 (1980) 
[4] Cooper-Taylor, C.: 50 ideas on using Twitter for education (2008), http: / / cooper-taylor.com/blog/2008/08/ 50-ideas-on-using-twitter-for-education/ (retrieved March 14, 2009)

[5] Costa, C., Beham, G., Reinhardt, W., Sillaots, M.: Microblogging in technology enhanced learning: A use-case inspection of ppe summer school 2008. In: Workshop on Social Information Retrieval for Technology Enhanced Learning (2008)

[6] EDUCAUSE Learning Initiative, 7 things you should know about... twitter, EDUCAUSE, Tech. Rep. (2007), http: / / connect. educause.edu/Library/ ELI/7ThingsYouShouldKnowAbout

[7] Fenner, A.-B.: Cultural awareness in the foreign language classroom. In: Cenoz, J., Hornberger, N.H. (eds.) Encyclopedia of Language and Education, 2nd edn., vol. 6, pp. 273285. Springer, Heidelberg (2008)

[8] Grosseck, G., Holotescu, C.: Can we use Twitter for educational activities? In: 4th International Scientific Conference eLSE eLearning and Software for Education (2008)

[9] Grosseck, G., Holotescu, C.: Indicators for the analysis of learning and practice communities from the perspective of microblogging as a provocative sociolect in virtual space. In: 5 th International Scientific Conference eLSE-eLearning and Software for Education (2009)

[10] Holotescu, C., Grosseck, G.: Using microblogging in education. case study: Cirip.ro. In: 6th International Conference on e-Learning Applications, Cairo, Egypt (January 2009)

[11] Honeycutt, C., Herring, S.C.: Beyond microblogging: Conversation and collaboration via twitter. In: HICSS 2009: Proceedings of the 42nd Hawaii International Conference on System Sciences, pp. 1-10. IEEE Computer Society, Washington (2009)

[12] Huberman, B.A., Romero, D.M., Wu, F.: Social networks that matter: Twitter under the microscope. First Monday 14(1) (2008)

[13] Hymes, D.H.: On communicative competence, pp. 5-26. University of Pennsylvania Press, Philadelphia (1971); extracts available in Brumfit, C.J. Johnson, K. (eds.) The communicative approach to language teaching, pp. 5-26. Oxford University Press, Oxford (1979)

[14] Java, A., Song, X., Finin, T., Tseng, B.: Why we twitter: understanding microblogging usage and communities. In: 9th WebKDD and 1st SNA-KDD 2007 workshop on Web mining and social network analysis, pp. 56-65. ACM, New York (2007)

[15] Kellogg, W.A.: Position paper: Social coherence in computer-mediated conversation. In: Discourse Architectures:Designing and Visualizing Computer Mediated Conversation. A workshop at CHI 2002 (2002)

[16] Krishnamurthy, B., Gill, P., Arlitt, M.: A few chirps about Twitter. In: WOSP 2008: First workshop on Online social networks, pp. 19-24. ACM, New York (2008)

[17] Lew, A.A.: Twitter tweets for higher education (2007), http: / /web20teach.blogspot.com/2007/08/ twitter-tweets-for-higher-education.html (retrieved March 14, 2009)

[18] Mischaud, E.: Twitter: Expressions of the whole self - an investigation into user appropriation of a web-based communications platform. Master's thesis, LSE - MEDIA@LSE (2007)

[19] Savignon, S.J.: Communicative Competence: Theory and Classroom Practice. McGrawHill Humanities/Social Sciences/Languages (1997)

[20] Shen, L., Shen, R.: The pervasive learning platform of a shanghai online college - a largescale test-bed for hybrid learning. In: Fong, J., Kwan, R., Wang, F.L. (eds.) ICHL 2008. LNCS, vol. 5169, pp. 178-189. Springer, Heidelberg (2008)

[21] Stevens, V.: Trial by Twitter: The rise and slide of the year's most viral microblogging platform. Teaching English as a Second or Foreign Language 12(1), 1-14 (2008) 